УДК 517.55

\title{
On a Class of A-Analytic Functions
}

\author{
Azimbai Sadullaev* \\ Nasridin M. Jabborov ${ }^{\dagger}$ \\ National University of Uzbekistan \\ Vuzgorodok, Tashkent, 100174 \\ Uzbekistan
}

Received 10.05.2016, received in revised form 06.06.2016, accepted 01.07.2009

We consider A-analytic functions in case when $A$ is anti-holomorphic function. In paper for A-analytic functions the integral theorem of Cauchy, integral formula of Cauchy, expansion to Taylor series, expansion to Loran series, Picard's big theorem and Montel's theorem are proved.

Keywords: A-analytic function, integral theorem of Cauchy, integral formula of Cauchy, Taylor series, Loran series, Picard's big theorem, Montel's theorem.

DOI: 10.17516/1997-1397-2016-9-3-374-383.

\section{Introduction and preliminaries}

Quoting from a well-known American mathematician Lipman Bers [1]: "It would be tempting to rewrite history and to claim that quasiconformal transformations have been discovered in connection with gas-dynamical problems. As a matter of fact, however, the concept of quasiconformality was arrived at by Grötzsch [2] and Ahlfors [3] from the point of view of function theory". The present work is devoted to the theory of analytic solutions of the Beltrami equation

$$
f_{\bar{z}}(z)=A(z) f_{z}(z)
$$

which directly related to the quasi-conformal mappings. The function $A(z)$ is, in general, assumed to be measurable with $|A(z)| \leqslant C<1$ almost everywhere in the domain $D \subset \mathbb{C}$ under consideration. Solutions of equation (1) are often referred to as $A$-analytic functions in the literature.

The solutions of equation (1), as well as quasi-conformal homeomorphisms in the complex plane $\mathbb{C}$ have been studied in sufficient details. Here we confine ourselves by giving the references $[1,3-13]$ and formulating the following three theorems:

Theorem 1.1 (see [3]). For any measurable on the complex plane $\mathbb{C}$ function $A(z):\|A\|_{\infty}<1$ there exists unique homeomorphic solution $\chi(z)$ of the equation (1) which fixes the points 0,1 and $\infty$.

Note that if the function $|A(z)| \leqslant C<1$ is defined only in the domain $D \subset \mathbb{C}$, then it can be extended to the whole $\mathbb{C}$ by setting $A \equiv 0$ outside $D$, so the Theorem 1.1 holds for any domain $D \subset \mathbb{C}$.

\footnotetext{
*sadullaev@mail.ru

†jabborov61@mail.ru

(c) Siberian Federal University. All rights reserved
} 
Theorem 1.2 (see $[5,6])$. The set of all generalized solutions of equation (1) is exhausted by the formula $f(z)=\Phi[\chi(z)]$, where $\chi(z)$ is a homeomorphic solution from Theorem 1.1, and $\Phi(\xi)$ is a holomorphic function in the domain $\chi(D)$. Moreover, if the generalized solution $f(z)$ has isolated singular points, the holomorphic function $\Phi=f \circ \chi^{-1}$ also has isolated singular points of the same types.

From Theorem 1.2 implies that the $A$-analytic function $f$ carries out internal mapping, i.e. it mapping an open set to an open set. It follows that the maximum principle holds for such functions: for any bounded domain $D \subset \mathbb{C}$ the maximum of modulus is reaches only on the boundary, i.e. $|f(z)|<\max _{z \in \partial D}|f(z)|, z \in D$. If the function is not zero, then the minimum principle also holds, i.e. $|f(z)|>\min _{z \in \partial D}|f(z)|, z \in D$.

Theorem 1.3 (see [8]). If a function $A(z)$ belongs to the class of m-smooth functions $(A(z) \in$ $C^{m}(D)$ ), then every solution $f$ of the equation (1) also belongs to, at least, the class, i.e. $f \in$ $C^{m}(D)$.

The purpose of this paper is to study $A$-analytic functions in a particular case, when the function $A(z)$ is anti-holomorphic in a considered domain. As we can see below, in this special case the solution of (1) possesses many properties of analytic functions, has an excellent integral representation, and can be expanded into Taylor, Laurent series and etc.

The considered case of $A$-analytic functions was initiated with a number of their applications in mechanics, geology and medicine, particularly, in the problems of tomography: X-ray, seismic, etc. They are associated with the Radon problem of recovery of functions from the given properties on the hyperplanes. In a series of papers A. L. Buhgeym and S. G. Kazantsev [14] Radon problem is interpreted by boundary problems for the infinite-dimensional analogue of the equation $f_{\bar{z}}-A f_{z}=0$, where $f$ is complex argument function of $z$, with values in some Banach space $X$ and $A$ is a linear continuous operator $A: X \rightarrow X,\|A\|<1$.

$A$-analytic functions can be applied to the theory of elliptic equations (see [15-17]), when $A$ is a continuous linear operator in finite or infinite-dimensional spaces. In papers [15-17] $A$ is a linear continuous operator in $X$. In the case, when $X=\mathbb{C}$ it is a constant, i.e. $A=$ const.

\section{Construction of Cauchy kernel when $\partial A=0$}

Let $A$ is anti-analytic, $\partial A=0$ in $D \subset \mathbb{C}$ such that $|A(z)| \leqslant C<1, \forall z \in D$. We put

$$
\begin{aligned}
& D_{A}=\frac{\partial}{\partial z}-\bar{A}(z) \frac{\partial}{\partial \bar{z}}, \\
& \bar{D}_{A}=\frac{\partial}{\partial \bar{z}}-A(z) \frac{\partial}{\partial z} .
\end{aligned}
$$

Then according to (1) the class of $A$-analytic functions $f \in O_{A}(D)$, characterized by the fact that $\bar{D}_{A} f=0$. Since, anti-analytic function is infinitely smooth, then from Theorem 1.3 implies that $O_{A}(D) \subset C^{\infty}(D)$.

Theorem 2.1. (analogue of Cauchy's theorem [16]). If $f \in O_{A}(D) \cap C(\bar{D})$, where $D \subset \mathbb{C}$ is a domain with rectifiable boundary $\partial D$, then

$$
\int_{\partial D} f(z)(d z+A(z) d \bar{z})=0
$$


Proof of the theorem follows directly from Stoke's formula:

$$
\begin{gathered}
\int_{\partial D} f(z)(d z+A(z) d \bar{z})=\int_{\partial D} f(z) d z+\int_{\partial D} f(z) A(z) d \bar{z}= \\
=\iint_{D} d f(z) \wedge d z+\iint_{D} d[f(z) A(z)] \wedge d z= \\
=\iint_{D} \frac{\partial f(z)}{\partial \bar{z}} d \bar{z} \wedge d z+\iint_{D}\left(\frac{\partial f(z)}{\partial z} A(z)+f(z) \frac{\partial A(z)}{\partial z}\right) d z \wedge d \bar{z}= \\
=\iint_{D}\left(-\frac{\partial f(z)}{\partial \bar{z}}+\frac{\partial f(z)}{\partial z} A(z)\right) d z \wedge d \bar{z}=0 .
\end{gathered}
$$

Now we assume that the domain $D \subset \mathbb{C}$ is convex and $\xi \in D$ its fixed point. We consider the function

$$
K(z, \xi)=\frac{1}{2 \pi i} \cdot \frac{1}{z-\xi+\overline{\int_{\gamma(\xi, z)} \bar{A}(\tau) d \tau}}
$$

where $\gamma(\xi, z)$ is a smooth curve which connects the points $\xi, z \in D$. Since the domain is simply connected and the function $\bar{A}(z)$ is holomorphic, then the integral $I(z)=\int_{\gamma(\xi, z)} \bar{A}(\tau) d \tau$ does not depend on a path of integration; it coincides with a primitive, i.e. $I^{\prime}(z)=\bar{A}(z)$.

Theorem 2.2. $K(z, \xi)$ is A-analytic function outside of the point $z=\xi$, i.e. $K \in O_{A}(D \backslash\{\xi\})$. Moreover, at $z=\xi$ the function $K(z, \xi)$ has a simple pole.

Proof. A simple check shows that the function

$$
\psi(z, \xi)=z-\xi+\overline{I(z)}=z-\xi+\overline{\int_{\gamma(\xi, z)} \bar{A}(\tau) d \tau}
$$

is $A$-analytic in $D$ :

$$
\frac{\partial}{\bar{z}}[z-\xi+\overline{I(z)}]=\frac{\partial}{\bar{z}} \overline{I(z)}=\overline{\frac{\partial}{\partial z} I(z)}=\bar{A}(z)=\bar{A}(z) \frac{\partial}{z}[z-\xi+\overline{I(z)}],
$$

i.e. $\psi(z, \xi) \in O_{A}(D)$.

The function $\psi(z, \xi)=z-\xi+\overline{\int_{\gamma(\xi, z)} \bar{A}(\tau) d \tau}$ has a unique simple zero at the point $z=\xi$. In fact, if $[\xi, z]$ is a segment which connects the points $\xi, z \in D$, then

$$
z-\xi+\overline{\int_{\gamma(\xi, z)} \bar{A}(\tau) d \tau}=z-\xi+\overline{\int_{[\xi, z]} \bar{A}(\tau) d \tau}
$$

and since $|A(z)| \leqslant c<1$, we have

$$
\begin{gathered}
\mid z-\xi+\overline{\int_{\gamma(\xi, z)} \bar{A}(\tau) d \tau|\geqslant| z-\xi|-| \int_{[\xi, z]} \bar{A}(\tau) d \tau|\geqslant| z-\xi\left|-\int_{[\xi, z]}\right| A(\tau)|| d \tau \mid \geqslant} \\
\geqslant|z-\xi|-c \cdot \int_{[\xi, z]}|d \tau|=(1-c)|z-\xi|>0, \quad z \neq \xi .
\end{gathered}
$$

This implies that the function $\psi(z, \xi)$ has only one zero at the point $z=\xi$, and this zero is simple. This means that $K(z, \xi)$ is holomorphic in $D \backslash\{\xi\}$ and the point $z=\xi$ is its simple pole. This proves the theorem. 
Remark 2.3. If the domain $D \subset \mathbb{C}$ is not a convex, but only simply connected, then although the function $\psi(z, \xi)$ is uniquely defined in the $D$, but a priori, it might has the other isolated zeros except $\xi: \psi(z, \xi)=0, z \in P=\left\{\xi, \xi_{1}, \xi_{2}, \ldots\right\}$. Consequently, $\psi \in O_{A}(D), \psi(z, \xi) \neq 0$ when $z \notin P$ and $K(z, \xi)$ is analytic function only in $D \backslash P$, it has a poles at the points of $P$. Due to this fact, we consider the class of $A$-analytic functions only in the convex domain $D \subset \mathbb{C}$.

According to Theorem 1.2, the function $\psi(z, \xi) \in O_{A}(D)$ carries out an internal mapping. In particular, the set

$$
L(\xi, r)=\left\{z \in D:|\psi(z, \xi)|=\left|z-\xi+\overline{\int_{\gamma(\xi, z)} \bar{A}(\tau) d \tau}\right|<r\right\}
$$

is open in $D$. For sufficiently small $r>0$ it compactly belongs to $D$ and contains the point $\xi$. This set is called $A$-lemniscate with center $\xi$ and denoted by $L(\xi, r)$. According to the maximum principle the lemniscate $L(\xi, r)$ is simply connected and to the minimum principle it is connected.

It is clear that $K(z, \xi) \in L_{l o c}^{1}(D), \forall \xi \in D$ and a differential form of first degree $\omega=$ $K(z, \xi)(d z+A(z) d \bar{z})$ is determines a current by the formula

$$
\omega \circ \alpha=\int \omega \wedge \alpha, \alpha \in F^{1}(D)
$$

where $F^{1}(D)$ is class of finite, infinitely smooth differential forms of first degree.

Theorem 2.4. A differential of the current is coincide with the Dirac measure $\delta_{\xi}$, i.e. for any finite infinitely smooth in $D$ function $\varphi \in F^{0}(D)$ we have

$$
d \omega \circ \varphi=\int \omega \wedge d \varphi=\varphi(\xi), \alpha \in F^{0}(D) .
$$

Proof. We have

$$
\begin{gathered}
d \omega=d K \wedge(d z+A d \bar{z})= \\
=d K \wedge d z+d(K \cdot A) \wedge d \bar{z}=\frac{\partial K}{\partial \bar{z}} d \bar{z} \wedge d z+A \cdot \frac{\partial K}{\partial z} d z \wedge d \bar{z}=0, z \in D \backslash\{\xi\} .
\end{gathered}
$$

Hence,

$$
\int \omega \wedge d \varphi=\lim _{\varepsilon \rightarrow 0} \int_{D \backslash L(\xi, \varepsilon)} \omega \wedge d \varphi=\lim _{\varepsilon \rightarrow 0} \int_{D \backslash L(\xi, \varepsilon)} d(\omega \varphi)=\lim _{\varepsilon \rightarrow 0} \int_{\partial L(\xi, \varepsilon)} \omega \varphi, \quad \alpha \in F^{0}(D) .
$$

On the other hand, since $d \psi(z, \xi)=d z+d \bar{I}(z)=d z+A(z) d \bar{z}$, then

$$
\int_{\partial L(\xi, \varepsilon)} \omega=\int_{\partial L(\xi, \varepsilon)} K(z)(d z+A(z) d \bar{z})=\frac{1}{2 \pi i} \int_{|\psi(z, \xi)|=\varepsilon} \frac{d \psi(z, \xi)}{z-\xi+\int_{\gamma(\xi, z)} \bar{A}(\tau) d \tau}=\frac{1}{2 \pi i} \int_{|\psi|=\varepsilon} \frac{d \psi}{\psi}=1
$$

and

$$
\int \omega \wedge d \varphi=\lim _{\varepsilon \rightarrow 0} \int_{\partial L(\xi, r)} \omega \varphi=\varphi(\xi), \quad \alpha \in F^{0}(D)
$$

Theorem 2.5 (Cauchy formula [18]). Let $D \subset \mathbb{C}$ is an arbitrary convex domain and $G \subset D$ is a subdomain, with piecewise smooth boundary $\partial G$. Then for any function $f(z) \in O_{A}(G) \cap C(\bar{G})$ we have a formula

$$
f(z)=\int_{\partial G} K(\xi, z) f(\xi)(d \xi+A(\xi) d \bar{\xi}), \quad z \in G
$$


Proof. Fix a point $z \in G$ and a small circle $U(z, \varepsilon) \subset \subset G, \varepsilon>0$. Then by Theorem 2.1

$$
\int_{\partial G} K(\xi, z) f(\xi)(d \xi+A(\xi) d \bar{\xi})=\int_{|\xi-z|=\varepsilon} K(\xi, z) f(\xi)(d \xi+A(\xi) d \bar{\xi}) .
$$

But according to the Stoke's formula and Theorem 2.4 we have:

$$
\begin{gathered}
\int_{|\xi-z|=\varepsilon} K(\xi, z) f(\xi)(d \xi+A(\xi) d \bar{\xi})=\int_{|\xi-z|=\varepsilon} f(\xi) \omega(\xi, z)=\int_{|\xi-z| \leqslant \varepsilon} d[f(\xi) \omega(\xi, z)]= \\
=\int_{|\xi-z| \leqslant \varepsilon} d f(\xi) \omega(\xi, z)+\int_{|\xi-z| \leqslant \varepsilon} f(\xi) d \omega(\xi, z) \rightarrow 0+f(z)=f(z), \text { for } \varepsilon \rightarrow 0 .
\end{gathered}
$$

\section{The expansion of A-analytic functions to Taylor and Laurent series}

First we note that the analog power series for $A$-analytic functions will be

$$
\sum_{j=0}^{\infty} c_{j} \psi^{j}(z, a), \quad a \in D, \quad c_{j} \quad \text { is constant. }
$$

The domain of convergence of the series (4) is a lemniscate

$$
L(a, r)=\{|\psi(z, a)|<r\}
$$

where the radius of convergence is given by the Cauchy-Hadamard formula:

$$
\frac{1}{r}=\varlimsup_{j \rightarrow \infty} \sqrt[j]{\left|c_{j}\right|}
$$

There is true an inverse

Theorem 3.1. If $f(z) \in O_{A}(L(a, r)) \bigcap C(\bar{L}(a, r))$, where $L(a, r)=\{\xi \in D:|\psi(\xi, a)|<$ $r\} \subset \subset D$ is a lemniscate, then the function $f(z)$ can be expanded to the Taylor series in $L(a, r)$ :

$$
f(z)=\sum_{k=0}^{\infty} c_{j} \psi^{k}(z, a)
$$

where

$$
c_{k}=\frac{1}{2 \pi i} \int_{\partial L(a, \rho)} \frac{f(\xi)}{[\psi(\xi, a)]^{k+1}}(d \xi+A(\xi) d \bar{\xi}), \quad 0<\rho<r, \quad k=0,1, \ldots
$$

Proof. We fix $0<r^{\prime}<r$ and we write Cauchy formula for $L\left(a, r^{\prime}\right)$ :

$$
f(z)=\frac{1}{2 \pi i} \int_{|\psi(\xi, a)|=r^{\prime}} \frac{f(\xi)}{\psi(\xi, z)}(d \xi+A(\xi) d \bar{\xi}), \quad z \in L\left(a, r^{\prime}\right)
$$

Since

$$
\psi(\xi, z)=(\xi-a)-(z-a)+\overline{\int_{\gamma(a, \xi)} \bar{A}(\tau) d \tau}-\overline{\int_{\gamma(a, z)} \bar{A}(\tau) d \tau}=\psi(\xi, a)-\psi(z, a)
$$


then

$$
\begin{aligned}
& f(z)=\frac{1}{2 \pi i} \int_{|\psi(\xi, a)|=r^{\prime}} \frac{f(\xi)}{\psi(\xi, z)}(d \xi+A(\xi) d \bar{\xi})= \\
= & \frac{1}{2 \pi i} \int_{\partial L\left(a, r^{\prime}\right)} \frac{f(\xi)}{\psi(\xi, a)-\psi(z, a)}(d \xi+A(\xi) d \bar{\xi})= \\
= & \frac{1}{2 \pi i} \int_{\partial L\left(a, r^{\prime}\right)} \frac{f(\xi)}{\psi(\xi, a)\left[1-\frac{\psi(z, a)}{\psi(\xi, a)}\right]}(d \xi+A(\xi) d \bar{\xi}) .
\end{aligned}
$$

For $z \in L\left(a, r^{\prime}\right), \xi \in \partial L\left(a, r^{\prime}\right)$ we have $\left|\frac{\psi(z, a)}{\psi(\xi, a)}\right|<1$. Consequently,

$$
\frac{1}{1-\frac{\psi(z, a)}{\psi(\xi, a)}}=\sum_{k=0}^{\infty}\left[\frac{\psi(z, a)}{\psi(\xi, a)}\right]^{k} \text {. }
$$

Hence,

$$
\begin{gathered}
f(z)=\frac{1}{2 \pi i} \int_{\partial L\left(a, r^{\prime}\right)} \frac{f(\xi)}{\psi(\xi, a)\left[1-\frac{\psi(z, a)}{\psi(\xi, a)}\right]^{\prime}}(d \xi+A(\xi) d \bar{\xi})= \\
=\frac{1}{2 \pi i} \int_{\partial L\left(a, r^{\prime}\right)} \frac{f(\xi)}{\psi(\xi, a)} \sum_{k=0}^{\infty}\left[\frac{\psi(z, a)}{\psi(\xi, a)}\right]^{k}(d \xi+A(\xi) d \bar{\xi})= \\
=\sum_{k=0}^{\infty}\left\{\frac{1}{2 \pi i} \int_{\partial L\left(a, r^{\prime}\right)} \frac{f(\xi)}{[\psi(\xi, a)]^{k+1}}(d \xi+A(\xi) d \bar{\xi})\right\}[\psi(z, a)]^{k}=\sum_{k=0}^{\infty} c_{k}[\psi(z, a)]^{k},
\end{gathered}
$$

where $c_{k}=\frac{1}{2 \pi i} \int_{\partial L\left(a, r^{\prime}\right)} \frac{f(\xi)}{[\psi(\xi, a)]^{k+1}}(d \xi+A(\xi) d \bar{\xi})$. Since the function $\frac{f(\xi)}{[\psi(\xi, a)]^{k+1}}$ is $A$-analytic in the lemniscate $L(a, r)$, then by the Cauchy's theorem the path of integration $\partial L\left(a, r^{\prime}\right)$ can be changed to the arbitrary line $\partial L(a, \rho), 0<\rho<r$. This proves theorem.

Similarly, we prove

Theorem 3.2 (Laurent expansion). Let $f(z)$ be A-analytic in a ring of lemniscates: $f \in$ $O_{A}(L(a, R) \backslash L(a, r)), r<R$. Then $f(z)$ will be expanded to the Loran series in this ring:

$$
f(z)=\sum_{k=-\infty}^{\infty} c_{j} \psi^{k}(z, a)
$$

where a coefficients of Taylor-Laurent series which determines by the formula

$$
c_{k}=\frac{1}{2 \pi i} \int_{\partial L(a, \rho)} \frac{f(\xi)}{[\psi(\xi, a)]^{k+1}}(d \xi+A(\xi) d \bar{\xi}), \quad r<\rho<R, \quad k=0, \pm 1, \pm 2, \ldots
$$

The series (6) converges uniformly inside of the ring

$$
L(a, R) \backslash L(a, r)=\{z \in D: \quad r<|\psi(z, a)|<R\} .
$$

Cauchy inequality. For the coefficients of the Taylor-Laurent series holds the following inequalities

$$
\left|c_{k}\right| \leqslant \frac{\max \{|f(z)|: z \in \partial L(a, \rho)\}}{\rho^{k}}, r<\rho<R, \quad k=0, \pm 1, \pm 2, \ldots
$$


these inequalities easily follow from

$$
\int_{\partial L(a, \rho)}|d \xi+A(\xi) d \bar{\xi}|=2 \pi \rho .
$$

Laurent series (6) is a convenient tool to study isolated singularities of $A$-analytic function. If a point $a$ is an isolated singular point of the function $f$, i.e. $f \in O_{A}(0<|\psi(z, a)|<R)$, $R>0$, and if $f$ is bounded in some punctured neighborhood of $a$, then there is a finite number of in $(6) f(z)=\sum_{k=0}^{\infty} c_{j} \psi^{k}(z, a)$ and the point $a$ is a removable singular point of $f, f(a)=c_{0}$; if $\lim _{z \rightarrow a} f(z)=\infty$, then there are only finite number of terms of the series with negative indices $k$ in $(6)$ :

$$
f(z)=\sum_{k=-m}^{\infty} c_{j} \psi^{k}(z, a), m>0, c_{-m} \neq 0 .
$$

In this case, the point $a$ is called a pole of order $m$. In case, when $\lim _{z \rightarrow a} f(z)$ does not exists, $a$ called essential singular point.

\section{The big Picard's theorem and Montel's theorem}

In case, when $a$ essential singular point, there is a theorem of Sohodskiy: for any complex number $w$ there exists a sequence $z_{k} \rightarrow a: f\left(z_{k}\right) \rightarrow w$.

We note that according to Liouville's theorem anti-holomorphic function $A(z)$ which satisfies the condition $|A(z)| \leqslant c<1$ is constant in whole complex plane $\mathbb{C}$. Therefore, if we want to define an entire $A$-analytic function $f(z) \in O_{A}(\mathbb{C})$, we must request that $A(z) \equiv c,|c|<1$. For a such kind of entire $A$-analytic functions $f(z)$, the Picard's theorem holds: $A$-analytic functions $f \not \equiv$ const does not accept more than two points.

The next big Picard's theorem holds for an arbitrary anti-holomorphic in $D \subset \mathbb{C}$ function $|A(z)| \leqslant c<1$.

Theorem 4.1. If $a$ is an essential singular point of the A-analytic function $f(z)$, then $f$ does not accept more than two (exceptional) points of extended complex plane $\overline{\mathbb{C}}$.

In the proof of the theorem, by analogy of analytic functions, essentially used Montel's theorem on compact (normal) family of $A$-analytic functions and modular analytic function in the unit disk $U=U(0,1)$. Recall that the family of functions $\left\{f_{\alpha}(z)\right\}_{\alpha \in \Lambda} \subset O_{A}(G)$, is called normal if every its subfamily $\left\{f_{\alpha}(z)\right\}_{\alpha \in \Lambda_{0}}, \Lambda_{0} \subset \Lambda$ contains a subsequence

$$
\left\{f_{j}(z)\right\} \subset\left\{f_{\alpha}(z)\right\}_{\alpha \in \Lambda_{0}}: f_{j}(z) \rightrightarrows f(z), j \rightarrow \infty
$$

which uniformly converges inside of $G$.

Theorem 4.2 (Montel). A locally uniformly bounded family of A-analytic functions $\left\{f_{\alpha}(z)\right\}_{\alpha \in \Lambda} \subset O_{A}(G)$ forms a normal family.

To proof this theorem using Arzela's theorem it is enough to show equicontinuity of the family $\left\{f_{\alpha}(z)\right\}_{\alpha \in \Lambda}$ on any compact $K \subset \subset G$, that is $\forall \varepsilon>0 \quad \exists \delta>0: \forall z^{\prime \prime}, z^{\prime} \in K,\left|z^{\prime \prime}-z^{\prime}\right|<\delta, \forall f \in$ $\left\{f_{\alpha}\right\} \Rightarrow\left|f\left(z^{\prime \prime}\right)-f\left(z^{\prime}\right)\right|<\varepsilon$. 
Let $\rho=\max \{\sigma: L(w, \sigma) \subset G \forall w \in K\}>0$ and $K^{\rho / 2}=\bigcup_{w \in K} L(w, \rho / 2)$ - the blow-up of $K, K^{\rho / 2} \subset \subset G$, where $L(w, \sigma)-$ is lemniscate. Then $M=\sup \left\{\left\|f_{\alpha}\right\|_{K^{\rho / 2}}: \alpha \in \Lambda\right\}<\infty$ and according to expansion (5), for a $\delta<\rho$ the function

$$
\varphi_{\alpha}(z)=\frac{f_{\alpha}(z)-f_{\alpha}\left(z^{\prime}\right)}{\psi\left(z, z^{\prime}\right)}
$$

where $\psi\left(z, z^{\prime}\right)=z-z^{\prime}+\overline{\int_{\gamma\left(z^{\prime}, z\right)} \bar{A}(\tau) d \tau}$ is $A$-analytic function in the lemniscate $L\left(z^{\prime}, \rho\right) \subset \subset K^{\rho}$. Moreover,

$$
\left|\varphi_{\alpha}(z)\right| \leqslant \frac{2 M}{\rho / 2}=4 \frac{M}{\rho}, \quad z \in \partial L\left(z^{\prime}, \rho / 2\right)
$$

We note, that $z^{\prime \prime} \in L\left(z^{\prime}, \rho\right)$. By the maximum principle $\left|f_{\alpha}\left(z^{\prime \prime}\right)-f_{\alpha}\left(z^{\prime}\right)\right| \leqslant 4 \frac{M}{\rho}\left|\psi\left(z^{\prime \prime}, z^{\prime}\right)\right| \leqslant$ const $\left|z^{\prime \prime}-z^{\prime}\right|$, that means equicontinuity of the family $\left\{f_{\alpha}(z)\right\}_{\alpha \in \Lambda}$ on $K$.

Corollary 4.3. If each function of family of A-analytic functions $\left\{f_{\alpha}(z)\right\}_{\alpha \in \Lambda}$ do not accept two values $a \in \mathbb{C}, b \in \mathbb{C}, a \neq b$, then this family is normal.

In fact, considering the family $\left\{\frac{f_{\alpha}(z)-a}{b-a}\right\}_{\alpha \in \Lambda}$, we can assume that $a=0, b=1$. We use the modular function $\mu(z): U \rightarrow \mathbb{C} \backslash\{0,1\}$. Note that the modular function $\mu(z)$ conform maps the circular triangle $\Delta_{0}=A B C \subset \bar{U}, A, B, C \in \partial U$, with sides $A B \perp \partial U, B C \perp \partial U, C A \perp \partial U$ on the upper half of the plane so that $\mu(A)=0, \mu(B)=1, \mu(C)=\infty$. Then, it extends to the unit circle on the principle of symmetry: for example, a triangle $\Delta_{1}$, that is symmetrical triangle to $\Delta_{0}$ with respect the side $A B$ is translated to the lower plane. The inverse function $\mu^{-1}$ determines the multi-valued function with critical points $\{0,1, \infty\}$.

Now we fix a function $f_{\alpha}(z) \not \equiv$ const and a point $z^{0} \in G$ so that the image $f_{\alpha}\left(z^{0}\right)$ lies either in the upper half-plane or in the lower half-plane. We select in some small neighborhood $W$ of the point $f_{\alpha}\left(z^{0}\right)$ single-valued branch of the function $\mu^{-1}: W \rightarrow \Delta_{0} \cup \Delta_{1}$. Then the composition $\mu^{-1} \circ f_{\alpha}(z)$ is $A$-analytic function in a neighborhood $V \subset G$. But, since the function $\mu^{-1}$ has the critical point only in $\{0,1, \infty\}$, and $f_{\alpha}(z)$ does not accept these terms, then by the monodromy theorem, $\mu^{-1} \circ f_{\alpha}(z)$ extends to $G: \mu^{-1} \circ f_{\alpha}(z) \leqslant 1, z \in G$.

From theorem 4.2 implies that the family $\left\{\mu^{-1} \circ f_{\alpha}(z)\right\}_{\alpha \in \Lambda}$ and, therefore $\left\{f_{\alpha}(z)\right\}_{\alpha \in \Lambda}$ is a normal family.

Proof of theorem 4.1. Without loss of generality we may assume that $a=0$, i.e. 0 is essential singular point, $f(z): f \in O_{A}(\{|z|<\varepsilon\}), \lim _{z \rightarrow 0} f(z)$ does not exist. Assume the contrary: function $f$ accept two points $\{a, b\}$ in the disk $|z|<\varepsilon$. Take a sequence $\varepsilon=\varepsilon_{1}>\varepsilon_{2}>\ldots>\varepsilon_{j} \searrow 0$ and the ring $\frac{1}{4 \varepsilon_{j}}<|z|<\frac{1}{\varepsilon_{j}}, j=1,2, \ldots$ The family $\left\{f\left(\frac{z}{\varepsilon_{j}}\right)\right\}_{j}$ of $A$-analytic functions is normal in the ring $\frac{1}{4}<|z|<1$, because it accepts two values, $a \neq b$. According to Corollary 4.3 , it is normal, i.e., there is a sequence $\varphi_{k}(z)=f\left(\frac{z}{\varepsilon_{j_{k}}}\right)$, which uniformly converges inside $\frac{1}{4}<|z|<1$ to the function $F(z)$.

If $F(z) \equiv \infty$, then the sequence $\left\{\frac{1}{\varphi_{k}(z)-a}\right\}_{k}$ of $A$-analytic functions converges to zero on the circle $|z|=\frac{1}{2}$. It means that the function $d \frac{1}{f(z)-a}$ converges to zero on the circles $|z|=\frac{\varepsilon_{j_{k}}}{2}$, 
$k \rightarrow \infty$. By the maximum principle for $A$-analytic function $\frac{1}{f(z)-a}$ it follows

$$
\max \left\{\left|\frac{1}{f(z)-a}\right|: \frac{\varepsilon_{j_{k}}}{2} \leqslant|z| \leq \frac{\varepsilon_{j_{k+1}}}{2}\right\} \rightarrow 0
$$

Hence,

$$
\max \left\{\left|\frac{1}{f(z)-a}\right|: \frac{\varepsilon_{j_{k}}}{2} \leqslant|z|<0\right\} \rightarrow 0
$$

which means that $\lim _{z \rightarrow 0} f(z)=a$ which is impossible.

Now, if $F(z) \not \equiv \infty$, then $F(z)$ is $A$-analytic in the ring $\frac{1}{4}<|z|<1$. If we denote $M=$ $\max \left\{|F(z)|:|z|=\frac{1}{2}\right\}$, then $|f(z)| \leqslant 2 M,|z|=\frac{\varepsilon_{j_{k}}}{2}$ for sufficiently large $k$. By the maximum principle this inequality is true on the ring $\frac{\varepsilon_{j_{k}}}{2} \leqslant|z| \leqslant \frac{\varepsilon_{j_{k+1}}}{2}$, that is not possible, due to the essential singularity of the point 0 . This proves the theorem.

\section{References}

[1] L.Bers, Mathematical aspects of subsonic and transonic gas dynamics, Wiley, New York, 1958.

[2] H.Grötzsch, Über die Verzerrung bei schlichten nichtkonformen Abbildungen und über eine damit zusammenhängende Erweiterung des Picardschen Satzes, Ber. Verh. Sächs Akad. Wiss., 80(1928), 503-507.

[3] L.Ahlfors, Lectures on quasiconformal mappings, Princeton, N.J., Van Nostrand, 1966.

[4] P.P.Belinskiy, General properties of quasiconformal mappings, Moscow, Nauka, 1974, (in Russian).

[5] B.Bojarski, Homeomorphic solutions of Beltrami systems, Doklady Akademii Nauk SSSR, 102(1955), no. 4 , 661-664 (in Russian).

[6] B.Bojarski, Generalized solutions of a system of differential equations of the first order ofthe elliptic type with discontinuous coefficients, USSR Sbornik Mathematics, 43(85) (1957), 451-503.

[7] N.M.Jabborov, Kh.Kh.Imomnazarov, Some initial-boundary value probles in the mechanics of two-speed media, Tashkent, Uzbek. Natsionalny Universitet, 2012 (in Russian).

[8] I.N.Vekua, Generalized analytical functions, Moscow, Nauka, (1988 (in Russian).

[9] V.Gutlyanski, V.Ryazanov, U.Srebro, E.Yakubov, The Beltrami equation: a geometric approach, J. Math. Sci., 175(2011), 413-449.

[10] L.I.Volkovisskiy, Quasiconformal mappings, L'viv, 1954 (in Russian).

[11] L.Bers, An outline of the theory of pseudoanalytic functions, Bull AMS, 62(1956), no. 4 , 291-331. 
[12] M.A.Lavrentyev, B.V.Shabat, Methods of complex variable functions theory, Moscow, Fizmatgiz, 1958 (in Russian).

[13] S.L.Krushkal, R.Kyunau, Quasiconformal mappings - new methods and applications, Moscow, Nauka, 1984 (in Russian).

[14] A.L.Bukhgeym, Inversion formulas in inverse problems. Addition to the book of M. M. Lavrentyev and L. Ya. Savelyev "Linear operators and ill-posed problems" Moscow, Nauka, 1991, (in Russian).

[15] A.L.Bukhgeym, S.G.Kazantsev, Ellyptic systems of Beltrami type and tomography problems, Doklady Akademii Nauk SSSR, 315(1990), 15-19 (in Russian).

[16] E.V.Arbuzov, Cauchy problem for second order elliptic systems on the plane, Sib. Math. Journal, 44(2003), no. 1, 3-20.

[17] E.V.Arbuzov, A.L.Bukhgeym, Cauchy problem for A-harmonic funktions, Doklady RAN, 349(1996), no. 5 , 586-587 (in Russian).

[18] N.M.Jabborov, T.U.Otaboev, Integral formula for $A(z)$-analytic functions, Trudy Respublik. Konferentsii "Modern methods of mathematical physics and its applications", Tashkent, 2015 (in Russian).

[19] N.M.Jabborov, T.U.Otaboev, Cauchy theorem for $A(z)$-analytic functions, Uzbek Mat. Zhurn., (2014), no. 1, 15-18 (in Russian).

\title{
Об одном классе А-аналитических функций
}

\author{
Азимбай Садуллаев \\ Насридин М. Жабборов \\ Национальный университет Узбекистана \\ Вузгородок, 100174 \\ Узбекистан
}

$\overline{\text { Мь рассматриваем А-аналитические функиии в случае, когда } А \text { является антиголоморфной }}$ функиией. Для А-аналитических функций в статье доказываются интегральная теорема Коши, интегральная формула Коши, разложимость в ряд Тейлора и ряд Лорана, а также большая теорема Пикара и теорема Монтеля.

Ключевые слова: А-аналитическая функция, интегралъная теорема Коши, интегральная формула Коши, ряд Тейлора, ряд Лорана, большая теорема Пикара, теорема Монтеля. 